\title{
RISCOS E CONTROLES INTERNOS: UMA METODOLOGIA DE MENSURAÇÃO DOS NÍVEIS DE CONTROLE DE RISCOS EMPRESARIAIS*
}

\author{
INTERNAL RISKS AND CONTROLS: A METHODOLOGY \\ TO MEASURE BUSINESS RISK CONTROL LEVELS
}

\author{
WANDERLEI LIMA DE PAULO \\ Doutorando em Engenharia Elétrica com ênfase em Engenharia de Sistemas \\ pela Escola Politécnica da USP - Campus Capital \\ E-mail: wanderlei@riskoffice.com.br \\ FRANCISCO CARLOS FERNANDES \\ Professor Doutor do Programa de Pós-Graduação em Ciências Contábeis \\ da Fundação Universidade Regional de Blumenau - SC \\ E-mail: ffernandes@riskoffice.com.br
}

\author{
LUCIANA GAVAZZI BARRAGAN RODRIGUES \\ Professora Ms. do Curso de Ciências Contábeis \\ da Fundação Escola de Comércio Álvaro Penteado - SP \\ E-mail: Ibarragan@riskoffice.com.br \\ JORGE EIDT \\ Mestrando em Ciências Contábeis \\ pela Fundação Universidade Regional de Blumenau - SC \\ E-mail: jorgeeidt@hotmail.com
}

\section{RESUMO}

Este artigo apresenta uma metodologia de mensuração do nível de controle de riscos inerentes aos processos de qualquer entidade, a partir da construção de um índice de desempenho definido em função da capacidade dos controles mitigarem os riscos e da eficácia dos controles implantados. O modelo possibilita ao gestor de riscos identificar para quais riscos são necessárias ações de melhoria de controle, quais possuem níveis de controles adequados e quais controles em excesso, contribuindo, assim, para a otimização da aplicação dos recursos disponíveis para a gestão de riscos e controles internos nas empresas. A metodologia foi aplicada a um estudo de caso e mostrou-se satisfatória do ponto de vista de avaliação e identificação dos níveis de controle.

Palavras-chave: Controles Internos; Gestão de Riscos; Matriz de Riscos; Matriz de Importância-Desempenho.

\section{ABSTRACT}

This paper presents a control measurement methodology applied in the processes performed by any entity. The methodology is derived from the analysis of the mitigation capabilities of the controls currently in use by the entity. The method enables risk managers to identify which risks require urgent actions to improve the control levels, which risks are already adequately controlled and which ones are in a situation of excessive control. This kind of information can play an important role to improve the application of available internal risk and control management resources in companies. The methodology was applied to a case study and revealed to be a satisfactory tool to assess and identify control levels.

Keywords: Internal Controls; Risk Management; Risk Matrix; Importance-Performance Matrix. 


\section{APRESENTAC̣ÃO}

No contexto atual de gestão de riscos e controles internos nas empresas, uma estratégia utilizada é a de implementar ou aprimorar os controles internos com base na identificação e mensuração dos riscos empresariais (MARTIN et al., 2004; SPIRA, 2003; BERGAMINI JUNIOR, 2005). É possível considerar a existência de duas abordagens de mensuração de riscos, a qualitativa e a quantitativa (CROUHY; GALAl; MARK, 2004). Em ambas, a mensuração é definida a partir do conhecimento das variáveis freqüência (ou probabilidade de ocorrência) e severidade (ou impacto financeiro), associadas aos eventos de perdas identificados nos processos das empresas. Pela abordagem qualitativa, o nível de risco é avaliado a partir da atribuição de critérios de classificação à freqüência e à severidade, enquanto pela abordagem quantitativa o risco é avaliado por modelos probabilísticos (ver JORION, 2003; CRUZ, 2002). Neste artigo, considera-se a abordagem qualitativa.

Uma das técnicas empregadas para avaliação qualitativa de riscos é o processo de auto-avaliação conhecido como Control Self Assessment (CSA), que consiste em avaliar, de maneira descentralizada e contínua, a efetividade dos controles e a potencialidade (freqüência versus severidade) dos riscos, possibilitando a detecção de exposições indesejadas e a implementação de medidas corretivas (WADE; WYNNE, 1999). O processo de auto-gestão pode ser implantado através de dois métodos básicos: o mapeamento de processos (process mapping) e a aplicação de questionários (check lists) de controles internos (BCBS, 2003). O uso desses métodos tem produzido bons resultados no que se refere à identificação dos riscos que afetam as atividades empresariais, à avaliação dos níveis de exposição e à definição de planos de melhoria que conduzam a empresa a um ambiente de controle adequado. Por outro lado, tais métodos se mostram limitados quando se trata de detectar situações em que os controles implementados estejam aquém do necessário ou configurem um dispêndio excessivo em controles para aqueles riscos que não representam um potencial de perda relevante.

Há, portanto, um problema de otimização da relação entre o nível de controle desejado e os custos de implementação dos controles necessários. Considerações sobre a natureza desse problema são apresentadas pelo COSO (2004), que reconhece a existência de restrições de recursos, de tal forma que as empresas devem considerar os custos e os benefícios associados a cada alternativa de controle.

Nesse contexto, este artigo apresenta uma metodologia de mensuração do nível de controle de riscos, a partir da construção de um índice de desempenho de controle, composto pela capacidade de os controles mitigarem os riscos e pela eficácia dos controles implantados. A metodologia possibilita ao gestor de riscos identificar para quais riscos é necessário melhoria de controle, quais possuem controles adequados e quais controles em excesso. Para isso, definese uma matriz de desempenho de controle, a partir da qual é possível estabelecer regiões de prioridade de melhoria dos controles em função do nível de tolerância ao risco. A aplicação da metodologia viabiliza a alocação ótima dos recursos disponíveis para implantação de planos de ação para minimizar a exposição a riscos, à medida que auxilia o gestor de riscos a identificar o excesso de controles aplicados a riscos de baixo potencial de perda, e a ausência de controles para os riscos considerados prioritários.

Este artigo está organizado da seguinte forma: nas seções 2 e 3, são apresentadas, respectivamente, os conceitos de matriz de riscos e de matriz de importância-desempenho, a serem utilizados na elaboração da metodologia proposta. Na seção 4, apresenta-se a metodologia para a avaliação dos níveis de controle de riscos. A seção 5 apresenta uma aplicação da metodologia descrita na seção 4 . Algumas considerações finais são descritas na seção 6 .

\section{MATRIZ DE RISCOS}

A mensuração qualitativa de riscos pode ser gerada através de uma matriz, em que o nível de risco é definido pela composição das variáveis freqüência (probabilidade) e severidade (impacto financeiro), associadas aos eventos de perda (fatores de risco) inerentes ao processo avaliado (MARSHALL, 2002; CROUHY, GALAI e MARK, 2004). A matriz de riscos é uma ferramenta que pode ser empregada na análise de riscos de processos de várias naturezas. Exemplos de aplicações do conceito de matriz de riscos podem ser encontrados em MARTIN et al. (2004), HEWETT et al. (2004), FIGUEIREDO (2001), GARVEY e LANSDOWNE (1998) e BRASILIANO (2006).

Segundo BERGAMINI JUNIOR (2005), a tabulação dos riscos em uma matriz permite a clara e ordenada identificação dos riscos que podem afetar a empresa, tanto em termos de freqüência quanto de severidade. Em geral, adota-se uma classificação qualitativa para os níveis de freqüência e de impacto, que poderá variar em função do processo avaliado, do porte da empresa, do segmento de mercado de atuação da empresa, entre outros fatores (MARSHALL, 2002). Os Quadros $1 \bullet$ e $2 \bullet$ apresentam um exemplo de classificação de freqüência e de severidade, nos quais os valores utilizados para descrever os níveis de impacto financeiro (campo "Descrição") poderão ser definidos com base, por exemplo, no patrimônio líquido, no total de ativos ou em testes de materialidade.

A matriz de risco pode ser construída pela composição de pesos atribuídos às variáveis freqüência e severidade, podendo ser particionada em regiões que caracterizam os níveis de risco avaliados. A definição dessas regiões pode 


\begin{tabular}{|l|l|c|}
\hline \multicolumn{3}{|c|}{ Classificação de Freqüência por Evento } \\
\hline Classificação & \multicolumn{1}{|c|}{ Descrição } & Peso \\
\hline Raríssimo & Menos de uma vez por ano & 1 \\
\hline Raro & Uma vez por ano & 2 \\
\hline Eventual & Uma vez por semestre & 3 \\
\hline Freqüente & Uma vez por semana & 4 \\
\hline Muito Freqüente & Mais de uma vez por semana & 5 \\
\hline
\end{tabular}

Quadro 1 Exemplo de classificação e parametrização dos níveis de freqüência

\begin{tabular}{|l|r|r|c|}
\hline \multicolumn{4}{|c|}{ Classificação de Severidade por Evento } \\
\hline Classificação & \multicolumn{2}{|c|}{ Descrição } & Peso \\
\hline Perda muito baixa & $\mathrm{R} \$ 0,01$ & $\mathrm{R} \$ 500,00$ & 1 \\
\hline Perda baixa & $\mathrm{R} \$ 500,01$ & $\mathrm{R} \$ 5.000,00$ & 2 \\
\hline Perda média & $\mathrm{R} \$ 5.000,01$ & $\mathrm{R} \$ 50.000,00$ & 3 \\
\hline Perda alta & $\mathrm{R} \$ 50.000,01$ & $\mathrm{R} \$ 500.000,00$ & 4 \\
\hline Perda grave & $\mathrm{R} \$ 500.000,01$ & - & 5 \\
\hline
\end{tabular}

Fonte: Os autores

Quadro 2 Exemplo de classificação e parametrização dos níveis de impacto

variar em função do perfil de risco do gestor, dos processos avaliados e dos produtos e serviços operacionalizados. A Figura 10 ilustra um exemplo de matriz de riscos, com níveis de risco classificados em Baixo, Médio, Alto e Extremo. Nesse caso, as regiões de risco foram delimitadas com base nos valores das intensidades de risco, calculados pelo produto entre os pesos da variável freqüência e da variável severidade (ver campo "Peso" constante dos Quadros 1 e 2).

De modo geral, pode-se considerar que os riscos situados na região de alto risco seriam indicativos de necessidade de controles mais rígidos, enquanto os riscos situados na região de baixo risco seriam um indicativo de controle adequado. Entretanto, essa leitura não pode ser generalizada a todos os tipos de riscos, dado que o nível de risco obtido a partir de uma matriz de risco não está diretamente associado à ausência ou excesso de controles. Por exemplo, a avaliação de um risco de oscilação do mercado de câmbio, em um cenário de alta freqüência e alta severidade, poderia situar-se na região de médio risco e estar no nível de controle desejável pelo gestor, desde que houvesse um elemento

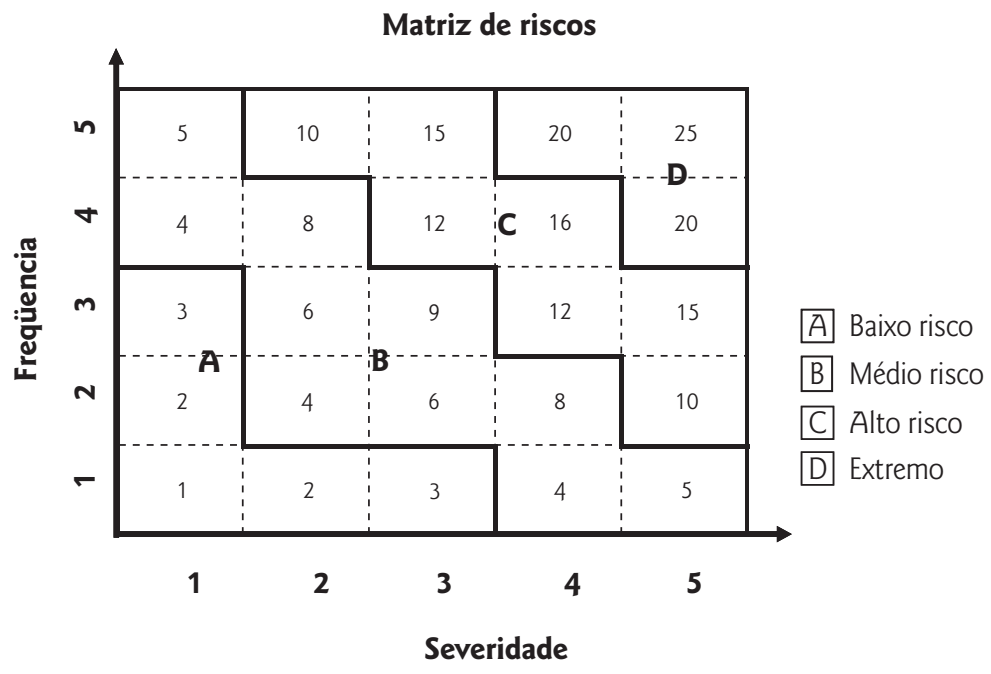

Fonte: Os autores

Figura 1 Exemplo de matriz de riscos 
mitigador, por exemplo, um hedge cambial que minimizaria ao menos o impacto financeiro de uma eventual perda, uma vez que o controle da freqüência está fora do domínio do gestor. Nessa situação, uma leitura direta da matriz de riscos poderia induzir à necessidade de implementação de novos controles quando não seriam necessários.

A metodologia proposta neste artigo contempla a mensuração dos níveis de controle e dos riscos, fornecendo uma alternativa para avaliação da capacidade de os controles adotados mitigarem os riscos, que pondera, através do estabelecimento de pesos, a relação custo-benefício implícita no processo de gestão de riscos e controles internos. O conceito de matriz de riscos será utilizado para definir o grau de importância dos riscos avaliados.

\section{MATRIZ DE IMPORTÂNCIA-DESEMPENHO}

Uma matriz de importância-desempenho é uma ferramenta presente no contexto de administração da produção de bens e serviços, normalmente utilizada para avaliar o desempenho de um produto ou de um processo produtivo (SLACK et al., 1997). Ela é construída a partir do nível de importância e do nível de desempenho de critérios competitivos associados aos produtos. Os critérios competitivos podem ser do tipo: rapidez e confiabilidade na entrega dos produtos; flexibilidade no projeto ou no mix de produção dos produtos e custo reduzido dos bens fabricados.

O nível de importância pode ser determinado por meio da classificação dos critérios em qualificadores e ganhadores de pedidos (HILL, 1994). Os níveis qualificadores representam uma determinada pontuação mínima necessária para competir no mercado. Os níveis ganhadores de pedido são aqueles nos quais o cliente se baseia para escolher seus fornecedores. A essas duas classificações podem-se acrescentar os níveis menos importantes que não são nem qualificadores e nem ganhadores de pedidos, não influenciando os clientes de forma significativa.

O nível de desempenho poderá ser julgado em função de ser melhor, igual ou pior do que o mercado. SLACK
(1994) propõe uma escala de nove pontos para mensurar o nível de importância e o nível de desempenho dos critérios competitivos. Após atribuir a pontuação relativa para cada critério competitivo, os resultados são plotados em uma matriz de importância-desempenho. Essa matriz pode ser dividida em quatro regiões de prioridade de melhoramento como mostra a Figura $2 \mathbf{0}$.

A região adequada é separada em sua margem inferior pela fronteira de aceitabilidade, sendo ela o nível mínimo de desempenho da empresa tolerável pelo mercado. Qualquer critério competitivo que cair na região de melhoramento é um candidato a ser aprimorado, porém se estiver no canto inferior esquerdo da matriz, poderá ser um caso não urgente de aprimoramento. A situação mais crítica é quando um critério competitivo encontra-se na região de ação urgente, exigindo, em curto prazo, a implementação de planos de melhoria. Por fim, existe, também, a região de excesso em que o desempenho atingido é superior ao necessário. Nesse caso, parte dos recursos poderia ser destinada à melhoria dos critérios situados na região de ação urgente.

Além da avaliação de processos produtivos, a matriz de importância-desempenho poderá ser utilizada para medir

\section{Matriz de Importância-Desempenho}

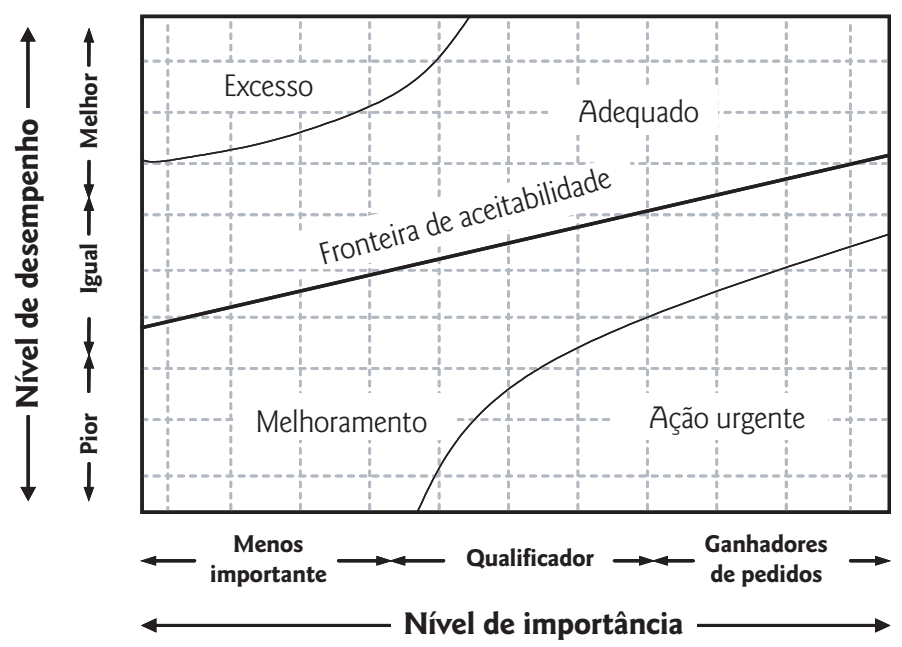

Fonte: Adaptado de SLACK, 1994

Figura 2 | Regiões de prioridade na matriz de importância-desempenho 
o desempenho de processos em empresas prestadoras de serviços (GIANESI; CORRÊA, 1996). Outros exemplos de aplicações da matriz de importância-desempenho podem ser encontrados em SELLITTO e WALTER (2006), PAULO (1999), GIANESI (1994), FALCÃO e MEDEIROS (2004) e SOUZA e ARBAGE (2001). Neste artigo, o conceito de ma- triz de importância-desempenho será utilizado na estruturação de uma matriz de desempenho que permita avaliar os níveis de controle e de riscos empresariais. Outra versão do conceito de matriz de importância-desempenho pode ser vista em MATZLER et al. (2004).

\section{METODOLOGIA PROPOSTA}

Esta seção apresenta a metodologia de mensuração do nível de controle de riscos, construída a partir da definição de um índice de desempenho de controle, composto pela capacidade dos controles mitigarem os riscos e pela eficácia dos controles implantados.

Considerem-se as seguintes definições,

$Q_{k}=\{1,2, \ldots, j, \ldots n\}$ : o conjunto de controles-padrão associado ao k-ésimo risco;

$A_{k j}=\{1,2, \ldots, m\}:$ o conjunto de atributos-padrão, associado ao j-ésimo controle do conjunto $Q_{k}$;

$U_{k}=\{1,2, \ldots, i, \ldots r\}:$ o conjunto de controles praticados, associado ao k-ésimo risco e

$H_{k, i}=\{1,2, \ldots l\}:$ o conjunto de atributos praticados, associado ao i-ésimo controle do conjunto $U_{k}$.

A diferenciação entre os conjuntos $Q$ e $U$, bem como entre $A$ e $H$ decorre do fato de que nem todo controle ou atributo padrão será praticado pela empresa.

Define-se o nível de controle de risco associado ao késimo risco, $N C R_{k}$, da forma

$$
N C R_{k}=\frac{\sum_{i \in U_{k}} w_{i} \alpha_{i}}{\sum_{j \in Q_{k}} w_{j}}
$$

em que $w_{i}$ e $w_{i}$ são os pesos relativos atribuídos ao i-ésimo controle praticado e ao j-ésimo controle-padrão, respectivamente. O peso $w$ representa o grau de capacidade dos controles estabelecidos mitigarem os riscos avaliados. $\mathrm{O}$ parâmetro $\alpha_{i}$ é dado por

$$
\alpha_{i}=\frac{\sum_{l \in H_{k i}} p_{l}}{\sum_{m \in A_{k j}} p_{m}}
$$

em que $p_{l}$ e $p_{m}$ são os pesos relativos atribuídos ao l-ésimo atributo praticado e ao m-ésimo atributo-padrão, respectivamente. O peso $p$ representa o grau de significância do atributo para a eficácia do j-ésimo controle na mitigação do k-ésimo risco. O Quadro 30 ilustra um exemplo de caracterização da capacidade de mitigação e de tipos de tributos de controle, com seus respectivos pesos.

O parâmetro $\alpha$ é um fator que penaliza o controle cujo conjunto de atributos praticados possui baixa significância para sua eficácia, comparado ao conjunto de atributos-padrão (o que equivale a $\alpha<1$ ). Por outro lado, a ausência ou o baixo poder de mitigação podem ser compensados quando o conjunto de atributos praticados é mais signifi-

\begin{tabular}{|c|c|c|c|c|c|}
\hline & Capacidade de Mitigação do Controle & $\begin{array}{l}\text { Peso do } \\
\text { Controle } \\
\qquad w\end{array}$ & & Tipos de Atributos de Controle & $\begin{array}{c}\text { Peso do } \\
\text { Atributo } \\
p\end{array}$ \\
\hline a) & $\begin{array}{l}\text { Após a aplicação do controle, o nível de } \\
\text { risco residual' está aquém das expectativas } \\
\text { da empresa. }\end{array}$ & 1 & a) & O controle permite testes periódicos. & 1 \\
\hline b) & $\begin{array}{l}\text { Após a aplicação do controle, o nível de } \\
\text { risco residual está em linha com as expecta- } \\
\text { tivas da empresa. }\end{array}$ & 2 & b) & $\begin{array}{l}\text { O controle corresponde a padrões } \\
\text { de boa prática referendados pelo } \\
\text { mercado. }\end{array}$ & 2 \\
\hline c) & $\begin{array}{l}\text { Após a aplicação do controle, o nível de } \\
\text { risco residual supera as expectativas da } \\
\text { empresa. }\end{array}$ & 3 & c) & $\begin{array}{l}\text { A aplicação do controle está for- } \\
\text { malmente estabelecida através de } \\
\text { normas e instruções escritas. }\end{array}$ & 3 \\
\hline
\end{tabular}
cativo que o conjunto de atributos-padrão (o que equivale

Fonte: Os autores

Quadro 3 Exemplo de classificação de capacidade de mitigação e de tipos de atributos de controle 


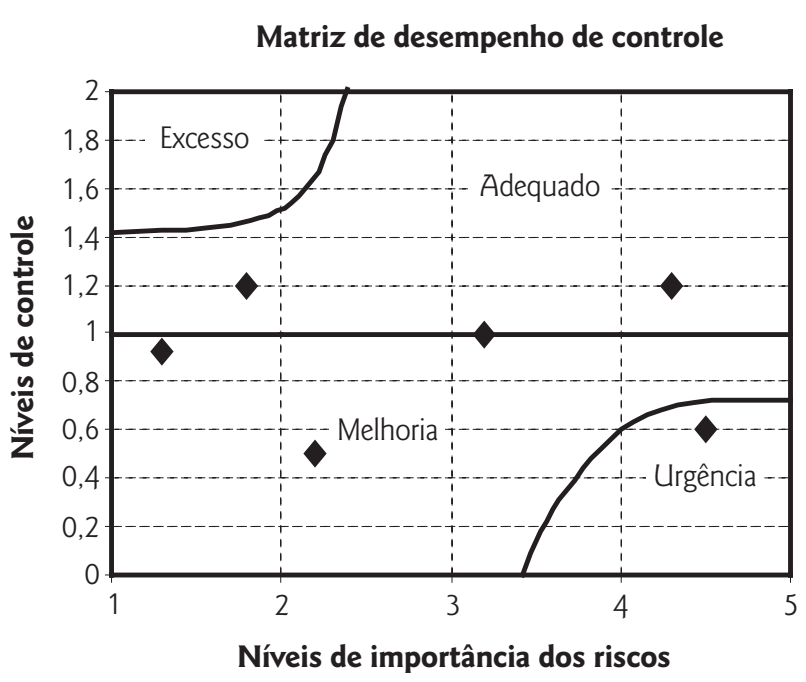

Fonte: Os autores

Figura 3 - Exemplo de uma matriz de desempenho de controle (matriz MDC)

a $\alpha>1$ ). Quando $\alpha=1$, o conjunto de atributos praticados equivale ao conjunto de atributos-padrão, atendendo, assim, ao grau de significância necessário para que o controle seja eficaz. Desse modo, $\alpha$ representa o quanto determinado controle implantado é eficaz.

O Nível de Controle de Riscos (NCR), definido pela relação (1), pode assumir os seguintes valores:

$N C R_{k}=1$
significa que o nível de controle é igual ao
padrão definido;

$N C R_{k}<1$ significa que o nível de controle está abaixo do padrão definido;

$N C R_{k}>1$ significa que o nível de controle está acima do padrão definido.

Dessa forma, é possível ao gestor identificar para quais riscos é necessária alguma melhoria de controle, quais possuem controle adequado e quais controles em excesso. Para viabilizar a alocação ótima dos recursos disponíveis para implantação de planos de ação para o controle de riscos, propõe-se a utilização de uma matriz de desempenho, aqui denominada Matriz de Desempenho de Controle (matriz MDC), gerada a partir do conceito de matriz de importânciadesempenho apresentado na Seção 3. Além da mensuração do nível de controle, a construção da matriz MDC requer a definição do nível de importância associado a cada risco. Considerando a escala de pesos para as variáveis freqüência
Matriz de desempenho de controle

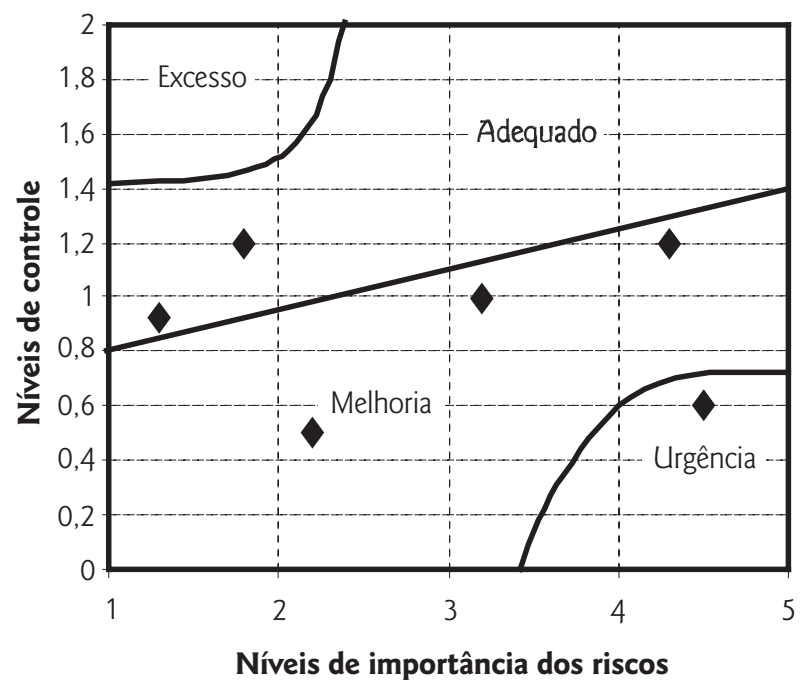

Fonte: Os autores

Figura 4 Exemplo de uma matriz de desempenho de controle com alteração na fronteira de aceitabilidade

e severidade estabelecida nos Quadros 1 e 2, o Nível de Importância de Riscos (NIR) pode ser escrito na forma

$$
N I R_{k}=1+\frac{\left(f_{k} I_{k}-1\right)}{6}, \operatorname{com} k \geq 1,
$$

em que $f_{k}$ e $I_{k}$ são, respectivamente, os pesos atribuídos à freqüência e à severidade do k-ésimo risco, conforme parâmetros definidos nos Quadros 1 e 2. Note-se que a relação (4) representa uma parametrização dos possíveis níveis de risco gerados a partir do conceito de matriz de riscos apresentado na Seção 2 , de modo que $1 \leq N I R_{k} \leq 5$. A Figura $3 \bullet$ mostra um exemplo de uma matriz MDC.

Note-se que, nesse caso, a fronteira de aceitabilidade equivale ao nível de controle-padrão (NCR $=1$ para qualquer nível de importância). As regiões de melhoria, de excesso, de urgência e de controle adequado, podem ser definidas pelo próprio gestor, mediante o grau de exigência de controle estabelecido. Por exemplo, a fronteira de aceitabilidade poderia ser definida considerando um valor mínimo de $N C R_{\min }=80 \%$ e um valor máximo de $N C R_{\max }=$ $140 \%$, conforme ilustra a Figura 4 ๑. Nesse caso, níveis de controle considerados adequados passam a ser inadequados mediante a alteração no padrão de aceitabilidade, caracterizando uma posição conservadora diante dos riscos mais significativos.

\section{ESTUDO DE CASO}

Essa seção apresenta um exemplo de aplicação da matriz MDC a um processo de gestão de contratos de uma
Entidade Fechada de Previdência Complementar (EFPC). No contexto atual de gestão de riscos nos fundos de pensão 
brasileiros, o uso da metodologia apresentada pode ser de grande valia, uma vez que as EFPC brasileiras passam por um período de grande atenção à gestão de riscos e aos controles internos (ver Resolução 13 do CGPC, 2004). O processo estudado é representado pelas atividades descritas na Figura $5 \mathbf{0}$.

A identificação dos riscos existentes no processo é o passo inicial para a construção da matriz MDC. Para que os riscos sejam identificados parte-se de um "dicionário" ou "catálogo" de riscos, nos quais são relacionadas as categorias e os tipos de riscos existentes no processo avaliado (CROUHY; GALAl; MARK, 2004). Para efeito de simplificação, será avaliada somente a atividade "Elaborar contratos", cujos riscos se limitam àqueles descritos no Quadro $4 \mathbf{0}$. Porém, outros tipos de riscos podem exis- tir no processo de elaboração de contratos, por exemplo, riscos de administração de contratos, riscos de falha humana ou riscos de sistemas, entre outros. Os pesos atribuídos às variáveis freqüência e severidade foram estabelecidos de acordo com as classificações apresentadas nos Quadros 1 e 2.

As definições de riscos apresentadas na coluna "Descrição" do Quadro 4 são as adotadas pela EFPC em que o estudo de caso foi realizado. Como para muitos riscos não há um padrão universalmente aceito, essas definições poderiam ser diferentes. Em linhas gerais, elas correspondem às apresentadas por MARSHALL (2002), conforme no Quadro 50.

O passo seguinte, na análise, é estabelecer para cada tipo de risco um conjunto de controles-padrão e para cada

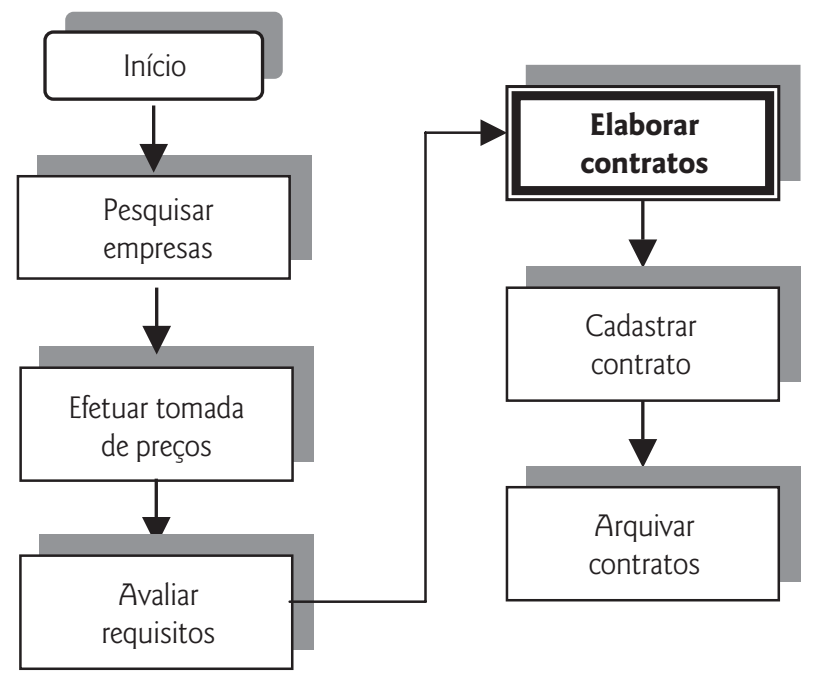

Fonte: Os autores

Figura 5 - Fluxo simplificado do processo de gestão de contratos

\begin{tabular}{|l|l|c|c|}
\hline \multirow{2}{*}{ Classificação dos Riscos } & \multicolumn{1}{c|}{ Descrição } & \multicolumn{2}{c|}{ Pesos atribuídos } \\
\cline { 2 - 4 } 1. Risco contratual & $\begin{array}{l}\text { Possibilidade de perda relacionada à inadequação formal do contrato, } \\
\text { à interpretação de suas cláusulas e à sua conformidade com a legisla- } \\
\text { ção pertinente. }\end{array}$ & 2 & \multicolumn{2}{c|}{ Impacto } \\
\hline $\begin{array}{l}\text { 2. Risco de concepção de } \\
\text { processos }\end{array}$ & $\begin{array}{l}\text { Possibilidade de perda ocasionada pela inadequação na concepção, } \\
\text { manutenção e comunicação dos processos negociais, de gestão e de } \\
\text { suprimentos. }\end{array}$ & 2 & 5 \\
\hline 3. Risco de conformidade & $\begin{array}{l}\text { Possibilidade de perda ocasionada pela inobservância, violação ou } \\
\text { interpretação indevida de regulamentos e normas. }\end{array}$ & 2 & 2 \\
\hline 4. Risco tributário & $\begin{array}{l}\text { Possibilidade de perda ocasionada por interpretação indevida da } \\
\text { legislação tributária. }\end{array}$ & 4 & 2 \\
\hline 5. Risco de terceirização & $\begin{array}{l}\text { Possibilidade de perdas decorrentes da transferência da gestão e } \\
\text { operação de processos internos para outras entidades. }\end{array}$ & 2 & 2 \\
\hline
\end{tabular}

Fonte: Os autores

Quadro 4 || Classificação dos tipos de riscos, dos impactos financeiros e das freqüências de ocorrências referentes à atividade elaborar contratos 


\begin{tabular}{|l|l|}
\hline Definição adotada pela EFPC & Definição correspondente em MARSHALL (2002) \\
\hline Risco Contratual & Risco Jurídico ou Legal (p. 423-424) \\
\hline Risco de Concepção de Processos & Risco de Projeto (p. 270) \\
\hline Risco de Conformidade & Risco de Regulamentação - Quebra de Conformidade (p. 430-431) \\
\hline Risco Tributário & Risco Tributário (p. 431) \\
\hline Risco de Terceirização & Risco de Fornecedor (p. 437) \\
\hline
\end{tabular}

Fonte: Os autores

Quadro 5 \Correspondência entre as definições de riscos adotadas pela EFPC e as apresentadas por MARSHALL (2002)

controle um conjunto de atributos-padrão. Por fim, atribuem-se pesos aos controles e aos seus respectivos atributos. Por necessidade de simplificação, tal procedimento será apresentado somente para o risco contratual. O
Quadro 60 apresenta uma relação de controles-padrão, de atributos e de seus respectivos pesos (esses últimos, de acordo com o Quadro 3). A descrição de cada atributo estabelecido é apresentada no Quadro $7 \mathbf{0}$.

\begin{tabular}{|c|c|c|c|c|}
\hline Riscos & Controles-padrão & Pesos $(w)$ & Atributos-padrão & Pesos $(p)$ \\
\hline \multirow{19}{*}{ 1. Risco contratual } & & & 1.1.1 & 2 \\
\hline & 1.1 Normas de Contratação & 3 & 1.1 .2 & 3 \\
\hline & & & 1.1 .3 & 2 \\
\hline & & & 1.1 .4 & 1 \\
\hline & & & 1.2 .1 & 3 \\
\hline & & & 1.2 .2 & 1 \\
\hline & & & 1.2 .3 & 3 \\
\hline & 1.2 Normas de Documentação & 2 & 1.2 .4 & 2 \\
\hline & & & 1.2 .5 & 2 \\
\hline & & & 1.2 .6 & 3 \\
\hline & & & 1.2 .7 & 2 \\
\hline & & & 1.3 .1 & 3 \\
\hline & 1.3 Práticas de Garantia de & 2 & 1.3 .2 & 1 \\
\hline & Conformidade & & 1.3 .3 & 2 \\
\hline & & & 1.3 .4 & 2 \\
\hline & & & 1.4 .1 & 3 \\
\hline & 1.4 Práticas de Gestão de & 2 & 1.4 .2 & 3 \\
\hline & Contratos & & 1.4 .3 & 2 \\
\hline & & & 1.4 .4 & 2 \\
\hline
\end{tabular}

Fonte: Os autores

Quadro 6 - Classificação dos tipos de controles, dos atributos e dos respectivos pesos

\begin{tabular}{|c|l|}
\hline $\begin{array}{c}\text { Atributo } \\
1.1 .1\end{array}$ & Descrição \\
\hline 1.1 .2 & $\begin{array}{l}\text { Existe um processo de revisão periódica, com participação de advogados, das minutas de contratos das transações celebradas? } \\
\text { da pertinência dos representantes legais quanto aos poderes necessários para celebrar o contrato e assumir as obrigações } \\
\text { nele contidas? }\end{array}$ \\
\hline 1.1 .3 & Há monitoramento das condições fiscais das pessoas físicas e jurídicas, nos casos de terceirização? \\
\hline 1.1 .4 & Há monitoramento para casos de terceirizações com possibilidade de perdas decorrentes de ações trabalhistas? \\
\hline 1.2 .1 & A instituição possui minutas de contratos adequadas para as transações que celebra? \\
\hline 1.2 .2 & $\begin{array}{l}\text { As normas asseguram que sejam adequadamente monitorados os contratos ou cláusulas configurados como exceções às } \\
\text { minutas? }\end{array}$ \\
\hline 1.2 .3 & $\begin{array}{l}\text { As normas e procedimentos formais garantem o envolvimento dos advogados na discussão de revisão de produtos ou } \\
\text { operações que já eram transacionados anteriormente mas que sofreram alterações por força de nova legislação? }\end{array}$ \\
\hline
\end{tabular}


(Continuação)

\begin{tabular}{|c|l|}
\hline Atributo & \multicolumn{1}{c|}{ Descrição } \\
\hline 1.2 .4 & $\begin{array}{l}\text { As normas e procedimentos escritos garantem o envolvimento de advogados na discussão de revisão ou criação de novos } \\
\text { produtos ou operações? }\end{array}$ \\
\hline 1.2 .5 & Existem check lists de documentos necessários para a celebração de cada tipo de contrato? \\
\hline 1.2 .6 & Existem normas e procedimentos que garantam a documentação das transações de forma tempestiva? \\
\hline 1.2 .7 & Os procedimentos asseguram que a documentação seja revisada? \\
\hline 1.3 .1 & Há processos formais de verificação de conformidade para as atividades sujeitas a regulamentações externas? \\
\hline 1.3 .2 & $\begin{array}{l}\text { Há sistemas ou bases de conhecimento que facilitam o acesso ao conteúdo das normas internas e regulamentos externos } \\
\text { que afetam os contratos celebrados? }\end{array}$ \\
\hline 1.3 .3 & $\begin{array}{l}\text { Os requisitos de conformidade com leis e regulamentos externos estão disponíveis para os funcionários executores das } \\
\text { atividades sujeitas a essas exigências? }\end{array}$ \\
\hline 1.3 .4 & $\begin{array}{l}\text { Os requisitos de conformidade com políticas e normas internas estão disponíveis para os funcionários executores das ativi- } \\
\text { dades sujeitas a essas exigências? }\end{array}$ \\
\hline 1.4 .1 & $\begin{array}{l}\text { As alterações de condições contratadas são tempestivamente documentadas e formalizadas com concordância das contra- } \\
\text { partes envolvidas, inclusive participantes ou assistidos da Entidade? }\end{array}$ \\
\hline 1.4 .2 & $\begin{array}{l}\text { As contrapartes (inclusive participantes e assistidos da Entidade) são comunicadas tempestivamente de eventuais altera- } \\
\text { ções em regulamentos de benefícios, cláusulas ou condições contratuais (preços pactuados, índices de correção, valores de } \\
\text { contribuições, benefícios, condições assistenciais)? }\end{array}$ \\
\hline 1.4 .3 & \begin{tabular}{l} 
Existem regras de proibição de discriminação contra fornecedores, participantes, funcionários ou colaboradores? \\
\hline 1.4 .4
\end{tabular} $\begin{array}{l}\text { Há documentos complementares (propostas comerciais, regulamentos de benefícios, glossários, modelos de avaliação de } \\
\text { desempenho de fornecedores etc.), referenciados ou anexados aos contratos, que auxiliem o entendimento das contrapar- } \\
\text { tes sobre os direitos e deveres estabelecidos nos contratos e nos planos de benefícios? }\end{array}$ \\
\hline 100
\end{tabular}

Fonte: Os autores

\section{Quadro 7 - Classificação dos atributos para cada controle associado ao riso de contrato}

O Quadro 80 apresenta o levantamento dos controles e atributos praticados, após um ciclo de avaliação, com- parados aos controles e atributos definidos como padrões para o risco contratual.

\begin{tabular}{|c|c|c|c|c|}
\hline Riscos & Controles-padrão & $\begin{array}{l}\text { Controles } \\
\text { praticados }\end{array}$ & Atributos-padrão & $\begin{array}{l}\text { Atributos } \\
\text { praticados }\end{array}$ \\
\hline \multirow{19}{*}{ 1. Risco contratual } & & & 1.1 .1 & $\sqrt{ }$ \\
\hline & 1.1 Normas de Contratação & $\sqrt{ }$ & 1.1 .2 & $\sqrt{ }$ \\
\hline & & & 1.1 .3 & \\
\hline & & & 1.1 .4 & \\
\hline & & & 1.2 .1 & $\sqrt{ }$ \\
\hline & & & 1.2 .2 & \\
\hline & & & 1.2 .3 & $\sqrt{ }$ \\
\hline & 1.2 Normas de Documentação & $\sqrt{ }$ & 1.2 .4 & $\sqrt{ }$ \\
\hline & & & 1.2 .5 & $\sqrt{ }$ \\
\hline & & & 1.2 .6 & $\sqrt{ }$ \\
\hline & & & 1.2 .7 & \\
\hline & & & 1.3 .1 & $\sqrt{ }$ \\
\hline & 1.3 Práticas de Garantia de & $\sqrt{ }$ & 1.3 .2 & $\sqrt{ }$ \\
\hline & Conformidade & & 1.3 .3 & $\sqrt{ }$ \\
\hline & & & 1.3 .4 & $\sqrt{ }$ \\
\hline & & & 1.4 .1 & $\sqrt{ }$ \\
\hline & 1.4 Práticas de Gestão de & $\sqrt{ }$ & 1.4 .2 & $\sqrt{ }$ \\
\hline & Contratos & & 1.4 .3 & $\sqrt{ }$ \\
\hline & & & 1.4 .4 & $\sqrt{ }$ \\
\hline
\end{tabular}

Fonte: Os autores

Quadro 8 EFPC - Levantamento dos controles e atributos praticados comparados ao padrão estabelecido 
O Quadro 90 descreve o conjunto-padrão de controle, $Q_{1}$, os conjuntos de atributos-padrão, $A_{1, j}$, $\operatorname{com} j=1, \ldots, 4$, o conjunto de controles praticados, $U_{1}$, e os conjuntos de atributos praticados, $H_{1,}$, com $i=1, \ldots, 4$, gerados a partir do levantamento apresentado no Quadro 80.

O mesmo procedimento descrito acima, utilizado para definir os conjuntos $Q, A$, $U$ e $H$, associados ao risco contratual, é aplicado aos demais riscos definidos no Quadro $4 \boldsymbol{0}$. Dessa forma, a Figura $6 \mathbf{O}$ apresenta a matriz de desempenho de controle resultante, cujos níveis de controle (NCR - eixo das ordenadas) e os níveis de importância dos riscos (NIR - eixo das abscissas) foram determinados através das equações (1) e (4), respectivamente. Note-se que a fronteira de aceitabilidade foi definida considerando um nível mínimo de controle $N C R_{\min }=70 \%$ e um nível máximo NCRmax $=120 \%$.

Pela Figura 6, nota-se que o risco contratual apresenta excesso de controle, de modo que parte dos recursos alocados em sua gestão poderia ser utilizada na melhoria do nível de controle dos riscos tributário e concepção de processo. Outra alternativa de realocação de recursos seria reduzir o nível de controle do risco de terceirização, dado que ele apresenta baixo nível de importância em relação aos demais riscos. Além da otimização de recursos, a matriz MDC permite avaliar o nível de controle e o risco residual entre dois ciclos de avaliação.

\begin{tabular}{|l|l|}
\hline Controles e Atributos-Padrão & \multicolumn{1}{|c}{ Controles e Atributos Praticados } \\
$Q_{1}=\{1.1,1.2,1.3,1.4\}$ & $U_{1}=Q_{1}$ \\
$A_{1,1}=\{1.1 .1,1.1 .2,1.1 .3,1.1 .4\}$ & $H_{1,1}=\{1.1 .1,1.1 .2\}$ \\
$A_{1,2}=\{1.2 .1,1.2 .2,1.2 .3,1.2 .4,1.2 .5,1.2 .6,1.2 .7\}$ & $H_{1,2}=\{1.2 .1,1.2 .3,1.2 .4,1.2 .5,1.2 .6\}$ \\
$A_{1,3}=\{1.3 .1,1.3 .2,1.3 .3,1.3 .4\}$ & $H_{1,3}=Q_{1,3}$ \\
$A_{1,4}=\{1.4 .1,1.4 .2,1.4 .3,1.4 .4\}$ & $H_{1,4}=Q_{1,4}$
\end{tabular}

Fonte: Os autores

Quadro 9 - Conjuntos de controles e atributos-padrão, e conjuntos de controles e atributos praticados

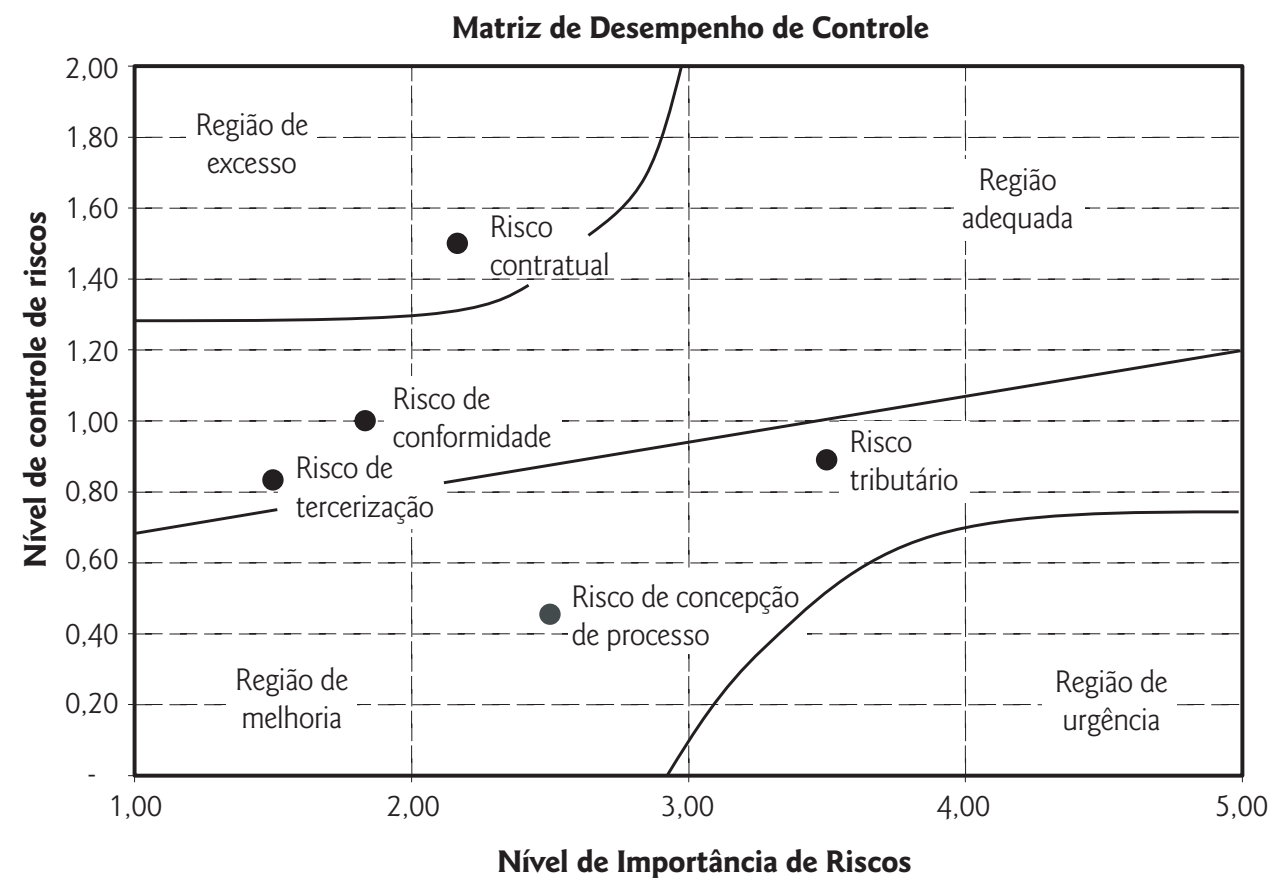

Fonte: Os autores

Figura 6 Matriz de desempenho de controles associada aos riscos inerentes à atividade elaborar contratos do processo de "gestão de contratos" da EFPC 


\section{CONSIDERAC̣ÕES FINAIS}

A metodologia apresentada neste artigo, caracterizada por uma matriz de desempenho de controle (matriz MDC), é uma ferramenta relevante no processo de mensuração e controle de riscos. Ela permite uma avaliação imediata do nível de desempenho dos controles existentes em uma instituição, oferecendo ao gestor de riscos condições melhores para o desempenho da tarefa de identificar para quais riscos são necessárias melhorias de controle, quais possuem controles adequados e quais controles em excesso.

Essa análise viabilizaria uma alocação mais adequada em termos de relação custo-benefício dos controles, contribuindo para a otimização do uso de recursos disponíveis para implantação de planos de mitigação de riscos. Isso ocorre à medida que o gestor obtém condições de reduzir os recursos aplicados a controles de riscos situados na região de excesso, ou próximos a ela, para aplicá-los a controles que necessitem de melhorias. Por exemplo, pela avaliação apresentada na Figura $6 \bullet$ um possível plano de ação seria alocar parte dos recursos destinados ao controle do risco contratual na melhoria do nível de controle do risco tributário.

Um ponto importante na utilização da matriz MDC é a definição das regiões de prioridade (regiões de excesso, de melhoria, de urgência e de controle adequado), dado que a avaliação final depende da delimitação de tais regiões. A princípio, as regiões seriam definidas a partir de critérios subjetivos, que caracterizariam o nível de exigência de controle estabelecido pelo gestor de riscos. Nesse sentido, uma possibilidade de melhoria da metodologia proposta neste artigo seria dada pela utilização de um procedimento sistemático para definir as regiões de prioridade.

\section{Referências Bibliográficas}

BASEL COMMITTEE ON BANKING SUPERVISION - BCBS. Sound Practices for the Management and Supervision of Operational Risk. BIS, 2003.

BERGAMINI JUNIOR, S. Controles Internos como um Instrumento de Governança Corporativa. Revista do BNDES,Rio de Janeiro, v.12, n.24, p.149-188, Dez., 2005.

BRASILIANO, A. C. R. Análise de Risco Corporativo., São Paulo: Sicureza Editora, 2006.

CONSELHO DE GESTÃO DA PREVIDÊNCIA COMPLEMENTAR. Resolução №. 13, de 10. de outubro de 2004. Estabelece princípios, regras e práticas de governança, gestão e controles internos a serem observados pelas entidades fechadas de previdência complementar - EFPC. Brasília, Ministério da Previdência e Assistência Social, 2004.

CROUHY, M.; GALAI, D.;MARK, R. Gerenciamento de Risco: Abordagem Conceitual e Prática: Uma Visão Integrada dos Riscos de Crédito, Operacional e de Mercado. Rio de Janeiro: Qualitymark, São Paulo: SERASA, 2004.

CRUZ, M. Modeling, Measuring and Hedging Operational Risk. John Wiley Trade, 2002.

FALCÃO, J. Filho N.; MEDEIROS, J. X. Análise da relação entre o produtor de ovos férteis e a agroindústria sob o aspecto tecnológico da produção: estudo de caso da empresa Asa Alimentos - DF. Trabalho apresentado ao Congresso ABIPTI 2004, disponível em http://intranet. planejamento.fiocruz.br/abpti/trabalhos/subtema01/t01.PDF, acesso em 10.02.2006

FIGUEIREDO, R. P. Gestão de Riscos Operacionais em Instituições Financeiras - uma abordagem qualitativa. 2001. Dissertação (Mestrado) - UNAMA, Belém - PA.

GARVEY, P. R., LANSDOWNE, Z. F.; Risk Matrix: An Approach for Identifying, Assessing, and Ranking Program Risks, Air Force Journal of Logistics, v. XXII, n. 1, June, 1998.

GIANESI, I. G. N. Gestão Estratégica de Serviços. 1994. Dissertação (Mestrado) - Escola Politécnica, Universidade de São Paulo, São Paulo - SP. ; CORRÊA, H.L. Administração Estratégica de Serviços: operações para a satisfação dos clientes. São Paulo: Atlas, 1996.

HEWETT, C.J.M.; QUINN, P.F.; WHITEHEAD, P.G.; HEATHWAITE, A.L.; FLYNN, N.J. Towards a nutrient export risk matrix approach to managing agricultural pollution at source. Hydrology and Earth System Sciences, v. 8, n. 4, p. 834-845, 2004.

HILL, T. Manufacturing Strategy: text and cases. Burr Ridge: Irwin, 1994.

JORION, P. Value at Risk - A Nova Fonte de Referência para o Controle de Risco de mercado. 2. ed. Editora BM\&F, 2003.

MARSHALL, C. L. Medindo e Gerenciando Riscos Operacionais em Instituições Financeiras. Rio de Janeiro: Qualitymark, 2002.

MARTIN, N.C.; SANTOS, L.R.; DIAS FILHO, J.M. Governança Empresarial, Riscos e Controles Internos: A Emergência de um Novo Modelo de Controladoria. Revista Contabilidade \& Finanças - USP, São Paulo, n. 34, p. 7-22, janeiro/abril 2004.

MATZLER, K.; BAILOM, F.; HINTERHUBER, H. H.; RENZL, B.; PICHLER, J. The asymmetric relationship between attribute-level performance and overall customer satisfaction: a reconsideration of the importance-performance analysis. Industrial Marketing Management, 33, pp. 271277, 2004.

PAULO, W. L. Análise da Avaliação da Produção na Pequena Empresa Têxtil. 1999. Dissertação (Mestrado) - EESC, Universidade de São Paulo, São Paulo. 
SELLITTO, M.A.; WALTER, C. Avaliação do desempenho de uma manufatura de equipamentos eletrônicos segundo critérios de competição. Produção, v.16, n. 1, p. 034-047, jan./abr. 2006.

SLACK, N. The Importance-Performance Matrix as a Determinant of Improvement Priority. International Journal of Operations and Production Management, v. 14, n. 5, p. 59-75, 1994.

; CHAMBERS, C.H.; HARRISON, A.; JOHNSTON, R. Administração da Produção. São Paulo: Atlas, 1997.

SOUZA, R.S.; ARBAGE, A. P. Processo de Formação de Estratégia: Um estudo de caso utilizando a matriz de Slack. Trabalho apresentado ao IV CONGRESSO INTERNACIONAL DE ECONOMIA E GESTÃO DE REDES AGROALIMENTARES, 2001, disponível em www.fearp.usp. br/egna/eng/year2001.htm, acesso em 10.02.2006.

SPIRA, L. F.; PAGE, M. Risk management: The reinvention of internal control and the changing role of internal audit. Accounting, Auditing and Accountability Journal, v. 16, n. 4, p. 640-661, 2003.

THE COMMITTEE OF SPONSORING ORGANIZATIONS OF THE TREADWAY COMMISSION - COSO. Enterprise Risk Management - Integrated Framework. New Jersey: AICPA, 2004.

WADE, K.; WYNNE, A. Control Self Assessment: For Risk Management and Other Practical Applications. John Wiley \& Sons, 1999.

\section{NOTA - Endereço dos autores}

Fundação Universidade Regional de Blumenau Rua Antônio da Veiga, 140 - Victor Konder Blumenau - SC

89012-900
Fundação Escola de Comércio Álvares Penteado Av. Liberdade, 532 - Liberdade

São Paulo - SP

01502-001 